\title{
Triphasic Waves Versus Nonconvulsive Status Epilepticus: EEG Distinction
}

\author{
Jean-Martin Boulanger, Charles Deacon, Diane Lécuyer, \\ Sylvie Gosselin, Jean Reiher
}

\begin{abstract}
Background: Triphasic waves (TWs) and generalized nonconvulsive status epilepticus (GNCSE) share morphological features that may create diagnostic ambiguity. Objective: To describe electroencephalographic differences between TWs and GNCSE. Methods: We retrospectively compared the electroencephalograms (EEGs) of two groups of patients presenting with decreased level of consciousness; those with TWs associated with metabolic encephalopathy and those with GNCSE. We studied the following: demographics, etiology and EEG morphological features. All EEGs were classified blindly (TWs or GNCSE) by two expert EEGers. Agreement between experts and concordance with clinical diagnosis were measured. Results: We analysed 87 EEGs (71 patients) with TWs and 27 EEGs (13 patients) with GNCSE. Agreement between experts and concordance with clinical diagnosis were excellent. When compared to TWs, epileptiform discharges associated with GNCSE had a higher frequency (mean $=2.4 \mathrm{~Hz} \mathrm{vs} 1.8 \mathrm{~Hz})(\mathrm{p}<0.001)$, a shorter duration of phase one $(\mathrm{p}=0.001)$, extra-spikes components $(69 \%$ vs $0 \%)(\mathrm{p}<0.001)$ and less generalized background slowing $(15.1 \%$ vs $91.1 \%)(\mathrm{p}<0.001)$. Amplitude predominance of phase two was common with TWs $(40.8 \%$ vs $0 \%)(\mathrm{p}=0.01)$. Lag of phase two was absent in all cases of GNCSE but present in 40.8\% of patients with TWs. Noxious or auditory stimulation frequently increased the TWs (51\%) while it had no effect on the epileptiform pattern ( $\mathrm{p}=0.008)$. Conclusions: Certain EEG morphological criteria and the response to stimulation are very helpful in distinguishing TWs from GNCSE.
\end{abstract}

RÉSUMÉ: Distinction électroencéphalographique : ondes triphasiques versus status épileptique généralisé nonconvulsif. Introduction: Les ondes triphasiques (OT) et le status épileptique généralisé nonconvulsif (SEGNC) peuvent être difficiles à distinguer sur l'EEG. Méthode: Nous avons analysé rétrospectivement les EEGs de deux groupes de patients: ceux avec OT dans un contexte d'encéphalopathie métabolique et ceux avec SEGNC. Tous les patients avaient une altération de la conscience. Les caractéristiques suivantes furent analysées: âge, étiologie sous-jacente et plusieurs caractéristiques morphologiques de l'EEG. Tous les EEGs ont été classifiés à l'aveugle (OT ou SEGNC) par deux électroencéphalographistes experts et l'exactitude par rapport au diagnostic clinique a été évaluée de même que la concordance entre les deux experts. Résultats: 87 EEGs (71 patients) avec OT et 27 EEGs (13 patients) avec SEGNC ont été analysés. La concordance entre les experts et l'exactitude de l'interprétation par rapport au diagnostic clinique étaient excellentes. Par rapport aux OT, les décharges épileptiformes associées au SEGNC avaient: une fréquence plus élevée (2,4 $\mathrm{Hz}$ vs $1,8 \mathrm{~Hz})(\mathrm{p}<0.001)$, une phase 1 de plus courte durée $(\mathrm{p}=0.001)$, des polypointes associées aux décharges $(69 \%$ vs $0 \%)(\mathrm{p}<0.001)$ et moins de ralentissement généralisé des rythmes de fond $(15,1 \%$ vs $91,1 \%)(\mathrm{p}<0.001)$. La prédominance d'amplitude de la phase 2 était fréquente avec les OT (40,8\% vs $0 \%$ ) ( $\mathrm{p}=0.01)$ de même qu'un délai de la phase 2 (40,8\% vs $0 \%)$. La stimulation douloureuse ou auditive causait une augmentation des OT $(51 \%)$ alors qu'elle n'avait aucun effet sur les anomalies épileptiformes. Conclusion: Certaines caractéristiques électrographiques morphologiques et l'effet de la stimulation sont très utiles pour distinguer les OT des anomalies épileptiformes du SEGNC.

Can. J. Neurol. Sci. 2006; 33: 175-180

The electroencephalogram (EEG) is a very useful tool for the evaluation of patients with altered levels of consciousness and coma. ${ }^{1,2}$ It permits one to rule out status epilepticus, confirm a diffuse or focal process and may orient the clinician towards an underlying etiology. Triphasic waves (TWs) on the EEG were described more than five decades ago and are usually attributed to a metabolic encephalopathy. ${ }^{3,4}$ The association of TWs with hepatic encephalopathy is well known. In the past, they were thought of as being pathognomonic of hepatic coma. We now know that TWs are associated with a wide variety of disorders (metabolic and non-metabolic) affecting the brain diffusely. 5,6

Triphasic waves are not epileptiform per se and are usually not associated with seizures. The TWs and generalized periodic or continuous epileptiform discharges occurring in generalized nonconvulsive status epilepticus (GNCSE) may share morphological features (Figures 1a, 1b, 1c). The second and third phases of TWs resemble the trough and wave of a spike wave. $^{7}$ According to Kaplan, ${ }^{8,9}$ differentiating these two electrographic entities may at times be very difficult. Both may disappear with benzodiazepines. ${ }^{10}$ The TWs can be falsely diagnosed as nonconvulsive status epilepticus and vice versa

From the Department of Neurology, Centre Hospitalier Universitaire de Sherbrooke, Quebec, Canada.

Received November 23, 2005. Accepted in final form February 13, 2006. Reprint requests to: Charles Deacon, Department of Neurology, Centre Hospitalier Universitaire de Sherbrooke, 3001 12th Avenue North, Sherbrooke, Quebec, J1H 5N4, Canada. 


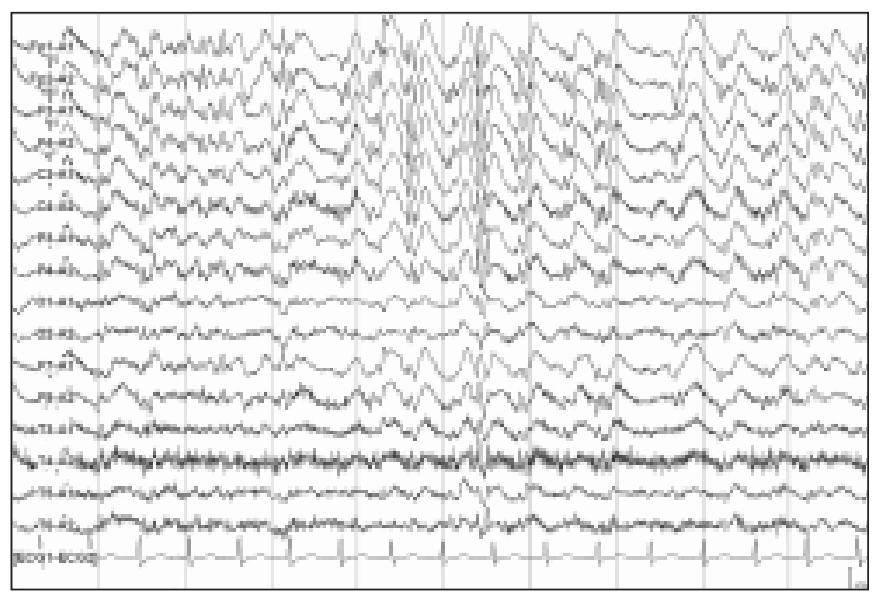

Figure 1a: The EEG of a patient with GNCSE showing generalized spike waves with many complexes having extraspikes (polyspikes).

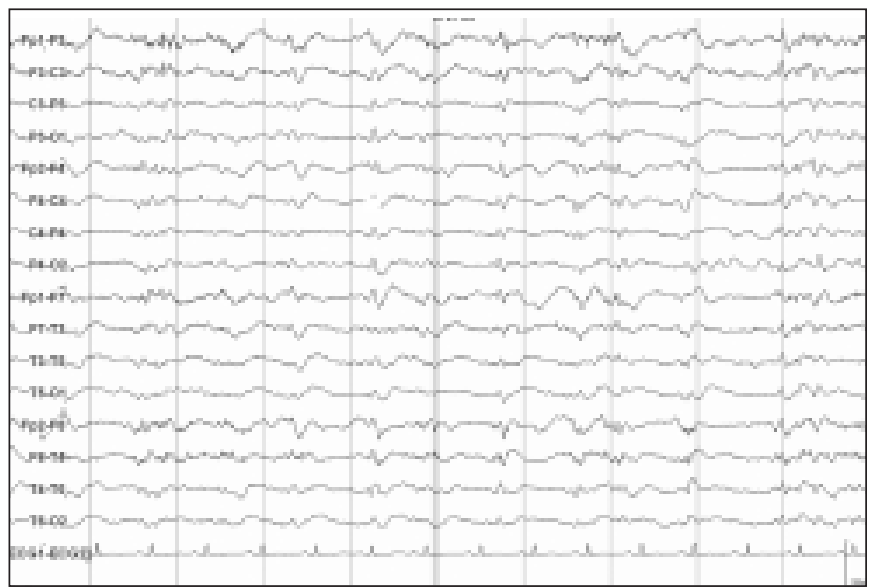

Figure 1b: This is an EEG from a 56-year-old patient presenting with GNCSE (absence status), showing periodic generalized spike waves without polyspikes.

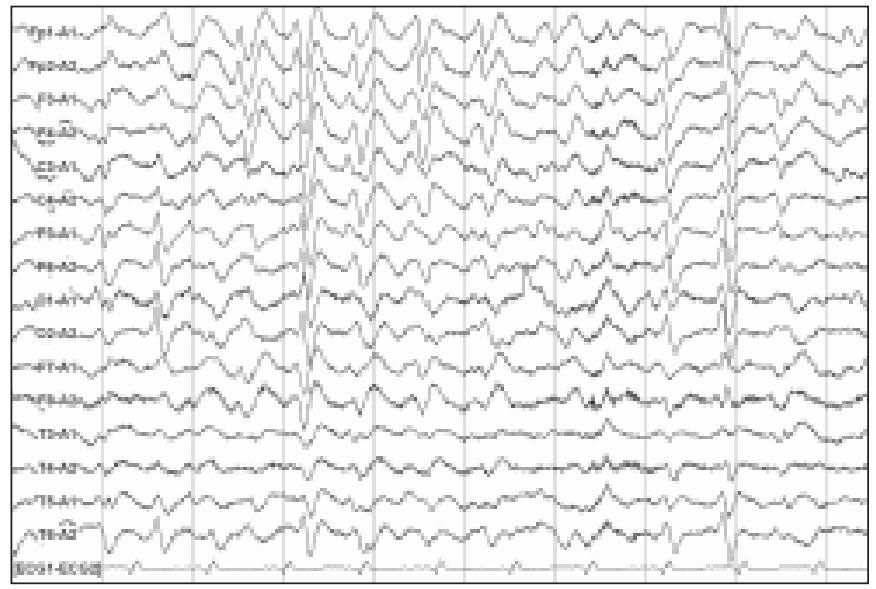

Figure 1c: The EEG of a patient with hepatic encephalopathy showing TWs. The first phase of many such complexes are apiculate and may look similar to generalized spike waves which are seen with GNCSE.
(Figure 2). In both conditions the patients will present with alteration of consciousness usually without obvious convulsive movements or clinical seizures. The management of the two conditions is very different (treating underlying metabolic encephalopathy for TWs and using benzodiazepines or other anticonvulsant drugs for GNCSE).

The presence of extraspike components (favoring GNCSE) and the presence of a positive or negative lag of the phase two (favoring TWs) may be helpful in differentiating the two entities. These features are not always present and may leave electrographic diagnostic ambiguity. Other potential differentiating characteristics include morphology (predominance of phase two), distribution (fronto-polar involvement), decreased quantity with sleep, all favoring TWs. ${ }^{11-13}$ These features have not been formally studied.

We decided to conduct a retrospective study on the

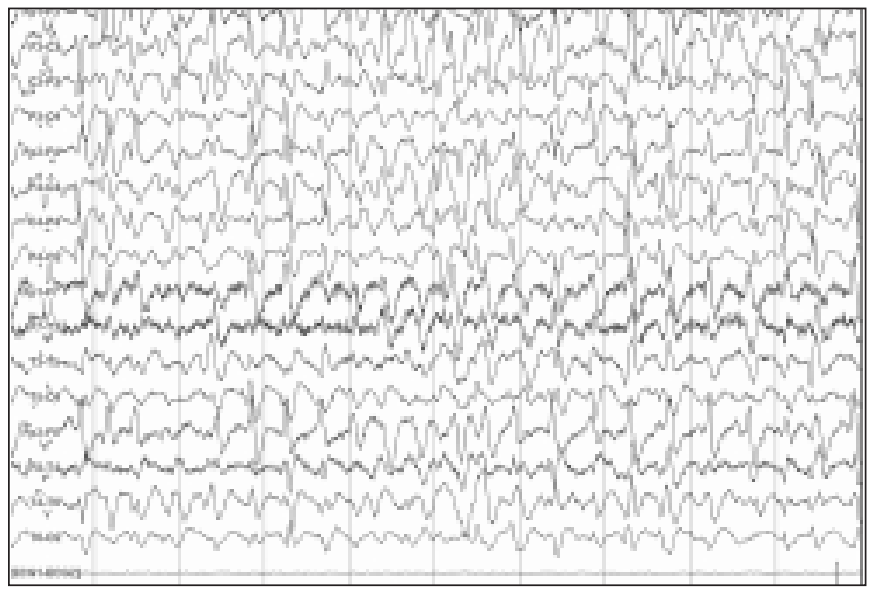

Figure 2: This EEG (longitudinal bipolar montage) of a 70-year-old female patient with hepatic cirrhosis, metabolic encephalopathy and TWs presenting with altered mental status was initially misdiagnosed as GNCSE. Note the spiky appearance of the first phase of each complex giving the false impression of spike wave discharges.

electrographic differentiation of TWs associated with metabolic encephalopathy and periodic or continuous generalized epileptiform discharges in the context of GNCSE, in patients evaluated with an EEG for altered consciousness.

\section{Methods}

\section{Case identification}

In this study, we retrospectively compared the EEGs of two groups of patients; individuals with definite TWs associated with metabolic encephalopathy and others with definite generalized periodic or repetitive epileptiform discharges in the context of GNCSE. The computerized database of the University of Sherbrooke EEG laboratory, containing results of 24552 EEGs performed from January $1^{\text {st }} 1997$ to December $1^{\text {st }} 2004$, was searched to identify cases of TWs and GNCSE. 


\section{Definitions of TWs and GNCSE}

We defined TWs as bilaterally synchronous bursts of complexes with three phases: negative (wave 1), positive (wave 2 ) and negative (wave 3 ). The duration of the complexes had to be between $300-600 \mathrm{msec}$. These discharges had to be recorded on patients with metabolic encephalopathy for which a cause was found.

We defined GNCSE as continuous or near-continuous generalized ictal discharges on the EEG associated with a mental status change from baseline for more than 30 minutes. ${ }^{14}$ This definition is not perfect but, so far, a clear and widely recognized clinical/EEG definition for nonconvulsive status epilepticus has not yet been established. ${ }^{15}$ With this broad definition, periodic generalized epileptiform discharges in the context of cerebral anoxia associated with coma were included. Ictal discharges included SW, repetitive sharp waves, sharp-and-slow waves or polyspikes and waves.

\section{EEG data}

The EEG tracings were recorded with a minimum of 18 electrodes and lasted a minimum of 20 minutes. All recordings included longitudinal bipolar, coronal and ipsilateral ear referential montages.

To ensure the validity of the two groups (TWs and epileptiform discharges) all EEGs were reviewed by two EEG experts while blinded to the clinical picture, diagnosis and original EEG report. Each record was classified separately by the two experts as containing epileptiform discharges, triphasic discharges or both. For each recording, if both blinded EEGexperts disagreeded with the original clinial-EEG diagnosis made by the attending medical team, this particular EEG was reclassified and switched from one group to the other (TWs group or GNCSE group). If there was disagreement between the blinded EEG-experts on the classification of a record, the original clinical-EEG diagnosis was used to settle the classification.

All selected EEG tracings were also reviewed to determine the quantity of discharges (more or less than 10 waves/100 seconds), mean frequency, distribution (exclusively anterior, diffuse or lateralized), electrodes of highest voltage and presence of a lag of phase two (anterior-posterior or posterior-anterior). Presence or absence of extra-spike(s) before phase one and generalized background slowing of the EEG activity were also noted. Relative amplitudes of individual waves in the complexes (TWs and epileptiform) were measured from the baseline before the discharges. A phase (wave) was considered dominant if its voltage exceeded other phases by more than $50 \%$. Phase one duration (in $\mathrm{msec}$ ) was also measured. The effects of sleep, stimulation (auditory, tactile or noxious) and benzodiazepines were also analyzed. The ipsilateral ear referential montage was used for measurements of relative amplitudes, electrodes of highest voltage, lag of phase two and distribution.

\section{Clinical data}

Hospital charts and EEG technologists' observations provided clinical diagnosis, level of consciousness at recording (confusion, stupor, coma), age, laboratory results and follow-up evolution (dead or alive).

\section{Statistical analysis}

Categorical variables were compared using the Fisher exact probability test. The Mann-Withney test was used for continuous variables. Kappa scores were calculated for concordance between the two experts (EEG reading) and for clinical diagnosis concordance. Two-sided $\mathrm{p}$ values less than 0.05 were considered to indicate significance. Data were analyzed with MedCalc statistical software.

\section{RESULTS}

\section{EEG data}

Between January 1st 1997 and December $1^{\text {st }} 2004$, a total of 24552 EEGs were performed. The TWs were described in 124 EEG reports (95 patients/0.51\% of EEGs) and periodic generalized epileptiform activity in 25 EEG reports (13 patients/0.10\%). In the TWs group 7 EEGs were excluded because they did not contain TWs and 30 EEGs were unavailable for analysis (either lost or destroyed). Therefore, we studied 87 EEGs (71 patients) with TWs and 25 EEGs (13 patients) with GNCSE.

\section{Inter-rater agreement and concordance with clinical picture}

The degree of concordance of each blinded electroencephalographer with the clinical diagnosis (TWs associated with metabolic encephalopathy or GNCSE) was very good with kappa scores of 0.91 (J.R.) and 0.84 (S.G.). Inter-rater agreement was also very good with a kappa score of 0.84 . Disagreement was observed only with 3 EEGs with TWs and 1 EEG with GNCSE. In the TWs group, 2 EEGs were rated by one expert as containing TWs and additional epileptiform discharges while they were rated as containing TWs only, by the other expert. The third EEG was rated GNCSE by one expert and TWs by the other. This EEG was recorded in a patient with sepsis and was labelled as containing TWs by the treating neurologist. In the GNCSE group, all EEGs were correctly labelled as containing epileptiform discharges but one EEG was identified as containing additional TWs by the two EEG experts. This EEG was recorded in a patient with altered consciousness following a single tonic-clonic seizure associated with sepsis related to an intra-abdominal abscess. Phenytoin was given and the patient's mental status improved the next day.

The classification of both groups of patients remained unchanged after this analysis. All of the records were in the correct category according to at least one EEG-expert.

\section{Demographics, mortality and etiology}

The mean age was 44.1 years (ranging from 24 to 73 ) for the GNCSE group compared to 70.1 years (ranging from 41 to 86 ) for the TWs group. The mortality rates were relatively high in both groups (59.1\% for TWs and $61.5 \%$ for GNCSE). Anoxia was the most frequent underlying etiology among those with GNCSE (seven out of 13 patients). All patients (four) with absence status and idiopathic generalized epilepsy survived. Clinical diagnoses and level of consciousness are shown for each group in Table 1.

In the TWs group, three patients $(4.2 \%)$ had acute generalized seizures within 24 hours of the recording. The underlying 


\section{Table 1: Clinical features and etiology of the two groups of patients (TWs and GNCSE)}

\begin{tabular}{|c|c|c|c|c|c|}
\hline Variables & \multicolumn{2}{|l|}{$\begin{array}{l}\text { TWs group, } \mathbf{n = 7 1} \\
(\%),+/ 2 \mathrm{SD}\end{array}$} & \multicolumn{2}{|l|}{$\begin{array}{l}\text { GNCSE group, } \mathbf{n = 1 3} \\
(\%),+/-2 \mathrm{SD}\end{array}$} & $\mathrm{P}$ \\
\hline Mean age & \multicolumn{2}{|l|}{$70.1+/-24.0$} & \multicolumn{2}{|l|}{$44.1+/-29.0$} & $<0.0001$ \\
\hline \multicolumn{6}{|l|}{ State } \\
\hline Confusion & \multicolumn{2}{|l|}{$22(31.0)$} & \multicolumn{2}{|l|}{$4(30.8)$} & 0.76 \\
\hline Stupor & \multicolumn{2}{|l|}{$38(53.5)$} & \multicolumn{2}{|l|}{$2(15.4)$} & 0.0246 \\
\hline Coma & \multicolumn{2}{|l|}{$11(15.5)$} & \multicolumn{2}{|l|}{$7(53.8)$} & 0.0064 \\
\hline \multicolumn{6}{|l|}{ Etiology } \\
\hline & Renal failure & $15(21.1)$ & Cerebral anoxia & $7(53.8)$ & \\
\hline & Hepatic failure & $11(15.5)$ & $\begin{array}{l}\text { Absence status with } \\
\text { IGE }\end{array}$ & $4(30.8)$ & \\
\hline & Multifactorial* & $15(21.1)$ & $\begin{array}{l}\text { Uncontrolled } \\
\text { symptomatic } \\
\text { epilepsy }\end{array}$ & $1(7.7)$ & \\
\hline & Cerebral anoxia & $7(9.8)$ & Sepsis & $1(7.7)$ & \\
\hline & Sepsis & $7(9.8)$ & & & \\
\hline & Delirium/dementia & $4(5.6)$ & & & \\
\hline & Other $^{\wedge}$ & $12(17.1)$ & & & \\
\hline
\end{tabular}

* Combination ( 2 or more) of hepatic, uremic, septic, toxic or anoxic encephalopathy. ${ }^{\wedge}$ Ischemic stroke (3 patients), intoxication with lithium (1) and codeine(1), subarachnoïd hemorrage (2), alcohol withdrawal (1), hypernatremia (2), hyponatriemia (1) and Bickerstaff encephalitis (1).

etiology for seizures in these patients were alcool withdrawal, subarachnoid hemorrage and recurrent seizures with probable epilepsy in the context of uremia. Another patient had a remote history of epilepsy related to a stroke but had no recent seizures. No additional epileptiform potentials were seen in these patients according to our blinded experts.

\section{EEG analysis}

Features that may permit electrographic differentiation between both groups of patients are shown in Table 2 .

Epileptiform discharges were of higher frequency $($ mean $=2.4 \mathrm{~Hz})$ compared to TWs $($ mean $=1.8 \mathrm{HZ}) \quad(\mathrm{p}<0.001)$. Phase one duration was longer in the TWs group than in the GNCSE group $(98.3 \mathrm{msec}$ vs $54.6 \mathrm{msec})(\mathrm{p}<0.001)$.

We were unable to find any significant difference in the electrode of highest voltage between both groups. The analysis of electrodes of highest voltage are shown in Table 3.

A lag of phase two was common in TWs group (29 patients or $40.8 \%$ ). It was never found in the GNCSE group ( $\mathrm{p}=0.01$ ). When present, the lag was mostly of the anterior-posterior type (20 EEGs or $68.9 \%$ ) and less commonly of the posterior-anterior type (9 EEGs or $31.1 \%$ ). The mean lag was respectively of $72.5 \mathrm{msec}+/-68.6(2 \mathrm{SD})$ and $-61.0 \mathrm{msec}+/-44.1(2 \mathrm{SD})$.

Phase two dominance (voltage $50 \%$ higher than other waves) was frequent among the TWs group (40.8\%) whereas it was never found in the GNCSE group ( $\mathrm{p}=0.01$ ). No dominant wave
Table 2: Electrographic differentiating features between patients with TWs $(\mathrm{N}=71)$ and patients with GNCSE $(\mathrm{N}=13)$

\begin{tabular}{|l|l|l|l|}
\hline Variables & TWs group & GNCSE group & P \\
\hline $\begin{array}{l}\text { Mean frequency of } \\
\text { discharges, Hz +/-2 } \\
\text { SD }\end{array}$ & $1.8+/-0.8$ & $2.4+/-1.0$ & $<0.0001$ \\
\hline Phase two lag (\%) & $29(40.8)$ & $0(0)$ & $0.279(\mathrm{NS})$ \\
\hline $\begin{array}{l}\text { Maximal } \\
\text { involvement of } \\
\text { frontopolar } \\
\text { electrode (\%) }\end{array}$ & $25(35.2)$ & $2(15.4)$ & $<0.0001$ \\
\hline $\begin{array}{l}\text { Extraspike } \\
\text { component (\%) }\end{array}$ & $0(0)$ & $9(69.0)$ & $<0.0001$ \\
\hline $\begin{array}{l}\text { Background } \\
\text { slowing (\%) }\end{array}$ & $65(91.5)$ & $0(0)$ & 0.01 \\
\hline $\begin{array}{l}\text { Amplitude } \\
\text { predominance of } \\
\text { phase two (\%) }\end{array}$ & $29(40.8)$ & $54.6+/-50.0$ & $<0.001$ \\
\hline $\begin{array}{l}\text { Phase 1 duration, } \\
\text { msec +/-2 SD }\end{array}$ & $98.3+/-27.4$ & $0(0)$ & 0.0022 \\
\hline $\begin{array}{l}\text { Increased amount } \\
\text { of discharges with } \\
\text { stimulation (\%) }\end{array}$ & $25\left(51.0^{*}\right)$ & & \\
\hline
\end{tabular}

* The proportion of patients where stimulation was performed

was the most frequent pattern in GNCSE (53.9\%) but was rarely seen with TWs $(5.7 \%)(\mathrm{p}<0.001)$ (Table 4). Extra-spikes were found in nine cases $(69.0 \%)$ with GNCSE and in none of those with TWs $(\mathrm{p}<0.001)$.

\section{Effect of benzodiazepines}

In the GNCSE group, benzodiazepines were given in eight out of 13 patients (61.5\%) and caused a marked decrease of epileptiform discharges in seven. The four patients with absence status associated with idiopathic generalized epilepsy had improvement in mental status while the three patients with anoxic encephalopathy remained comatose.

In the TWs group, benzodiazepines were administred only in two patients without significant electrographic or clinical change.

Table 3: Electrode of highest voltage in EEG recordings with TWs and GNCSE

\begin{tabular}{l|l|l|l|l|l|l}
\hline Group & \multicolumn{6}{|c}{ Electrode(s) of highest voltage } \\
\hline & None & Fp & F & C or P & Fp-F & \\
& Patients & Patients & Patients & Patients & Patients & \\
& $(\%)$ & $(\%)$ & $(\%)$ & $(\%)$ & $(\%)$ & \\
\hline TWs & 13 & 25 & 2 & 2 & 29 & 71 \\
& $(18.3 \%)$ & $(35.2 \%)$ & $(2.8 \%)$ & $(2.8 \%)$ & $(40.9 \%)$ & $(100 \%)$ \\
\hline GNCSE & 5 & 2 & 2 & 0 & 4 & 13 \\
& $(38.4 \%)$ & $(15.4 \%)$ & $(15.4 \%)$ & $(0 \%)$ & $(30.8 \%)$ & $(100 \%)$ \\
\hline
\end{tabular}

$\mathrm{Fp}=$ frontopolar, $\mathrm{F}=$ frontal, $\mathrm{C}=$ central, $\mathrm{P}=$ parietal 
Table 4: Dominant waves (more than $50 \%$ of other waves) in EEG recordings with TWs and GNCSE

\begin{tabular}{l|l|l|l|l|l|l}
\hline Group & \multicolumn{6}{|c}{ Dominant wave(s) } \\
\hline & None & 2,3 & 1,2 & 2 & 3 & \\
& Patients & Patients & Patients & Patients & Patients & \\
& $(\%)$ & $(\%)$ & $(\%)$ & $(\%)$ & $(\%)$ & \\
\hline TWs & 4 & 35 & 1 & 29 & 2 & 71 \\
& $(5.7 \%)$ & $(49.3 \%)$ & $(1.4 \%)$ & $(40.8 \%)$ & $(2.8 \%)$ & $(100 \%)$ \\
\hline GNCSE & 7 & 1 & 2 & 0 & 3 & 13 \\
& $(53.9 \%)$ & $(7.7 \%)$ & $(15.3 \%)$ & $(0 \%)$ & $(23.1 \%)$ & $(100 \%)$ \\
\hline
\end{tabular}

\section{Effect of sleep}

A minority of patients with TWs (8 out of 71) had a well defined sleep during their EEG. Sleep consisted mainly of diffuse theta activity without vertex waves or spindles. The triphasic complexes were abolished during that period. Sleep was not observed in the GNCSE group.

\section{Effect of stimulation}

Auditory and/or noxious stimulation was performed in 59 patients $(83.1 \%)$ with TWs and in all cases of GNCSE. In the TWs group, stimulation caused an immediate increase of triphasic discharges in 25 patients (51\%) (Figure 3) while stimulation had no effect on the epileptiform discharges in $\operatorname{GNCSE}(p=0.022)$.

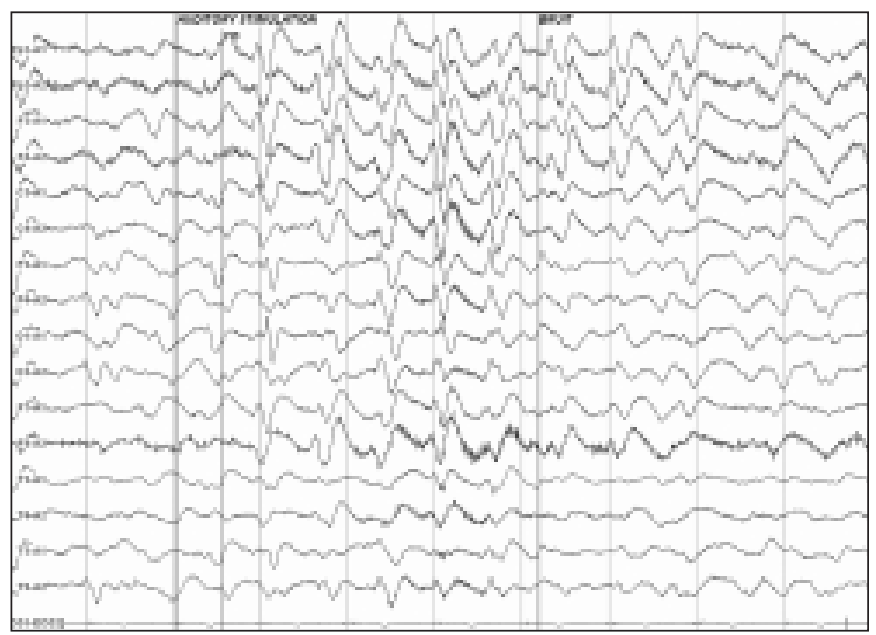

Figure 3: The EEG of a patient with altered consciousness with an increased amount of generalized complexes following auditory stimulation, suggestive of TWs.

\section{Discussion}

We conducted a retrospective study in order to better characterize the differences between TWs and periodic or continuous generalized epileptiform activity. All EEGs were performed at a single institution. Both conditions were seen relatively infrequently $(0.51 \%$ of EEGs for TWs and $0.10 \%$ for GNCSE). Nonetheless, TWs were five times more frequent than GNCSE in our patient population justifying the need to consider and exclude TWs before diagnosing GNCSE with an ambiguous EEG. Abundant and spiky TWs can be easily misdiagnosed as GNCSE especially when the underlying metabolic encephalopathy is not clinically appreciated.

We are not aware of any previous studies that attempted to differentiate these entities. This study, although retrospective, provides useful information. Some EEG morphological criteria may be of particular value. As was expected, lag of phase two and extraspike component were specific for TWs and GNCSE respectively. Other potential discriminating electrographic features included: phase one duration (longer in TWs), phase two dominance (TWs), frequency of discharges (more than $2 \mathrm{~Hz}$ in GNCSE) and background slowing (TWs). Of note, the ipsilateral ear montage was used for all the electrographic analysis and is strongly suggested for appreciation of TWs. In our view, longitudinal bipolar montage can be quite misleading in patients with TWs (Figure 2).

Response of waveforms to stimulation might be another useful way to discriminate between TWs and GNCSE. The TWs increased with stimulation, irregardless of the method used (auditory, visual, noxious) whereas GNCSE was not influenced by external stimuli. This interesting phenomenon suggests that TWs behave like sleep potentials, appearing when the level of consciousness is slightly increased with a stimulus and decreasing with deepening coma or more profound sleep.

In our study, sleep was rarely seen in the TWs group because of the short duration of the recording, retrospective design and intrinsic difficulties in identifying sleep in encephalopathy. Furthermore, sleep potentials (spindles, K-complexes and V waves) were not recognized in these patients. Amongst the few patients who had sleep, consisting of diffuse theta, TWs almost vanished during this time. A diminution of TWs with deepening sleep or coma has been described and could suggest that TWs have a thalamocortical origin as do sleep spindles and V waves. ${ }^{1,12}$ Still the effect of sleep on discharges might be of diagnostic value and a prospective study on that aspect should be undertaken.

Our two groups of EEGs were reviewed by two blinded EEGers with great knowledge and experience in the interpretation of EEG in various clinical settings. Very high concordance with the clinical data and between experts was obtained. These findings added validity to our groups avoiding selection bias.

The mortality rate was relatively high in both groups. The poor outcome was certainly related to the clinical diagnoses. For TWs, etiologies underlying metabolic encephalopathy were similar to other series. ${ }^{5,6}$ Uremic, hepatic and multifactorial encephalopathies were the most frequent. The GNCSE group was more heterogeneous with cerebral anoxia and absence status associated with generalized epilepsy being the most frequent causes. 
In this study, an anterior-posterior delay was found more commonly in TWs than a posterior-anterior delay. Sundaram and Blume $^{5}$ described opposite results in their series of TWs. The lag of phase two was best seen and calculated on ipsilateral ear referential montage.

Nine out of ten TWs EEGs $(91.5 \%)$ had a constant and important slowing of background activity while this was only the case in two EEGs of the GNCSE group. This finding could be explained by the common association between TWs and metabolic encephalopathy.

The TWs and GNCSE are sometimes difficult to distinguish from each other in patients with altered consciousness. In addition to morphological electrographic features, the way the discharges "behave" in response to stimulation might be very useful for distinction of the two entities.

\section{ACKNOWLEDGMENT}

The authors thank Marie-Pier Garand and Robert Black (Sherbrooke, Quebec, Canada) for providing statistical support for this paper.

\section{REFERENCES}

1. Bauer G. Coma and brain death. In: Niedermeyer E, Lopes Da Silva F, 4th ed. Electroencephalography: basic principles, clinical applications, and related fields. Baltimore:Williams and Wilkins; 1999. p.459-75.

2. Brenner R. The electroencephalogram in altered states of consciousness. Neurol Clin. 1985; 3(3): 615-31.
3. Foley JM, Watson CW, Adams RD. Significance of the electroencephalographic changes in hepatic coma. Trans Amer Neurol Ass. 1950; 75: 161-4.

4. Bickford RG, Butt HR. Hepatic coma: the electroencephalographic pattern. J Clin Invest. 1955; 34: 790-9.

5. Sundaram MBM, Blume WT. Triphasic waves: clinical correlates and morphology. Can J Neurol Sci. 1987; 14: 136-40.

6. Karnaze DS, Bickford RG. Triphasic waves: a reassessment of their significance. Electroenceph Clin Neurophysiol. 1984; 57: 193-8.

7. Nowack WJ, King JA. Triphasic waves and spike-wave stupor. Clin Electroencephalogr. 1992; 23(2): 100-4.

8. Kaplan PW. Prognosis in nonconvulsive status epilepticus. Epileptic Disord. 2000; 2: 185-93.

9. Kaplan PW. The EEG in metabolic encephalopathy and coma. J Clin Neurophysiol. 2004; 21: 307-18.

10. Fountain NB, Waldman WA. Effects of benzodiazepines on triphasic waves: implications for nonconvulsive status epilepticus. J Clin Neurophysiol. 2001; 18(4): 345-52.

11. Blume WT, Kaibara M, Young GB. Atlas of adult electroencephalography. 2nd ed. Philadelphia, 2002.

12. Baldy-Moulinier M, Besset A, Calvet B, Michel H. 24 hours polygraphic study of the waking-up and falling asleep periods in patients with hepatic encephalopathy. Rev Electroencephalogr Neurophysiol Clin. 1981; 11(1): 123-32.

13. Kennedy J, Parbhoo JP, MacGillivray BB, Sherlock S. Effect of extracorporeal liver perfusion on the electroencephalogram of patients in coma due to acute liver failure. Q J Med. 1973; 42: 549-61.

14. Shneker BF, Fountain NB. Assessment of acute morbidity and mortality in nonconvulsive status epilepticus. Neurology. 2003; 61: 1066-73.

15. Kaplan PW. Nonconvulsive status epilepticus. Neurology. 2003; 61: 1035-6. 